\title{
Ensayo de reproducción inducida en Ariopsis seemanni
}

\author{
Induced spawning trial with Ariopsis seemanni
}

\section{Ensajo de reprodução induzida em Ariopsis seemanni}

\author{
Víctor H. Espinel-Cárdenas ${ }^{1 *}$, Adriana Rodríguez-Forero ${ }^{2}$ \\ 1 Biólogo, Biología Marina, MSc, Departamento de Ciencias Naturales Exactas Universidad del Pacifico, Colombia \\ 2 Bióloga Marina, MSc, PhD, Programa de Ingeniería Pesquera, Grupo de Investigación y Desarrollo Tecnológico en \\ Acuicultura, Universidad del Magdalena, Colombia \\ Email: victorespinel@gmail.com
}

Recibido: 18 de octubre de 2016

Aceptado: 01 de agosto de 2018

\begin{abstract}
Resumen
El bagre marino Ariopsis seemanni es aprovechado como pez de consumo en la alimentación de pescadores y pobladores de la región pacifica colombiana y como pez ornamental (5 a $10 \mathrm{~cm}$-longitud total), a nivel comercial nacional e internacional. En Colombia esta especie ha sido priorizada en la agenda nacional de investigación en acuicultura de 2011-2012, como una de las especies marinas potenciales en actividades acuícolas. El objetivo de esta investigación fue evaluar el efecto de la gonadotropina coriónica humana (HGC) y el extracto pituitario de carpa (EPC) sobre la maduración final y el desove en hembras adultas de la especie. Se utilizaron 24 parejas de peces silvestres capturadas del medio, con un peso promedio de $263.6 \pm 42.2 \mathrm{~g}$ y $174.4 \pm 30.4 \mathrm{~g}$ para hembras y machos, respectivamente. Se utilizaron cuatro tratamientos hormonales: $\mathrm{T}_{1}: 5 \mathrm{mg}$ EPC/kg; T2: $7 \mathrm{mg}$ EPC/kg; T3: $2000 \mathrm{UI} \mathrm{HGC/kg} \mathrm{y} \mathrm{T4:} 1000 \mathrm{UI} \mathrm{HGC/kg}$. Se identificaron tres tipos de oocitos en maduración, no se presentaron diferencias estadísticamente significativas $(p>0,05)$ entre los tratamientos utilizados y la cantidad de oocitos en maduración; no se obtuvo desove espontaneo en ninguna de las hembras inducidas con las dosis hormonales empleadas.
\end{abstract}

Palabras clave: HGC; EPC; bagre; Canchimalo.

\begin{abstract}
The tete sea catfish Ariopsis seemanni is a fish used for human consumption by fishermen and residents along the coast of Colombia and as ornamental fish (5 to $10 \mathrm{~cm}$-total longitude), at national and international comercial level. In Colombia, this species has been prioritized in the national aquaculture research agenda 2011-2012, as one of the potential marine species for aquaculture activities. This research was aimed at evaluating the effect of human chorionic gonadotropin (HGC) and carp pituitary extract (EPC) on final maturation and spawning adult females of the species. 24 pairs of wild fish were used, with an average weight of $263.6 \pm 42.2 \mathrm{~g}$ and $174.4 \pm 30.4 \mathrm{~g}$ for females and males, respectively. Four hormonal treatments was be used: T1: EPC $5 \mathrm{mg} / \mathrm{kg} ; \mathrm{T}_{2}$ : EPC $7 \mathrm{mg} / \mathrm{kg} ; \mathrm{T}_{3}$ : HGC $2000 \mathrm{IU} / \mathrm{kg}$ and T4: HGC $1000 \mathrm{IU} / \mathrm{kg}$. Identify three types of maturation oocytes was. There were no statistically significant differences $(p>0,05)$ between treatments used and the number of oocytes achieving maturation. No spontaneous spawning was obtained in any of the females induced with the hormonal doses used.
\end{abstract}

Key words: HGC; EPC; Bagre; Canchimalo 


\begin{abstract}
Resumo
O bagre marino Ariopsis seemanni é aproveitado como peixe de consumo na alimentação de pescadores e moradores da região pacifica colombiana e como peixe ornamental (5 a $10 \mathrm{~cm}$ de comprimento), a nível comercial nacional e internacional. Na Colômbia A. seemanni tem sido priorizado na agenda nacional de pesquisa na aquicultura de 2011-2012, como uma das espécies marinhas potenciais em atividades aquícolas. Esta pesquisa teve como objetivo avaliar o efeito da gonadotrofina coriónica humana $(\mathrm{GCH})$ e o extrato pituitário de carpa (EPC) sobre a maduração final e o desove em fêmeas adultas desta espécie. Utilizaram-se 24 casais de peixes retirados do meio natural, com um peso médio de 263,6 \pm 42,2 g e 174,4 \pm 30,4 g para fêmeas e machos, respectivamente. Empregaram-se quatro tratamentos hormonais: T1: 5 mg EPC/kg; T2: 7 mg EPC/kg; T3: 2000 UI HGC/kg e T4: 1000 UI HGC/kg. Identificaram-se três tipos de oócitos em maduração, não houve diferencias estatisticamente significativas $(p>0,05)$ entre os tratamentos utilizados e a quantidade de oócitos em maturação; não foi possível obter desove espontâneo em nenhuma das fêmeas induzidas com as doses hormonais empregadas.
\end{abstract}

Palavras-chave: Bagre tete; Canchimalo; EPC; GCH; Tiburoncito.

\section{Introducción}

Desde el punto de vista de la supervivencia y perpetuación de las especies, la reproducción, puede ser considerado como un proceso fundamental de los organismos, por lo tanto, el control de los ciclos reproductivos de las especies ícticas sometidas a cultivo, es uno de los factores más importantes para asegurar el éxito de la acuicultura y constituye un requisito indispensable para la introducción de nuevas especies a los sistemas de producción (Cruz-Casallas et al., 2006).

Desde hace algunos años en Colombia se ha generado un interés por algunas especies de peces del orden de los bagres Siluriformes. Especies dulceacuícolas como Epseudoplatystoma magdaleniatum, anteriormente Pseudoplatystoma fasciatum (Bagre rayado), Leiarius marmoratus ((Yaque), Pimelodus grosskopffi (Capaz), Phractochphalus hemioliopterus (Cajaro), Sorubim cuspicaudus (Blanquillo), Pimelodus blochii (Nicuro), CaIlophysus macropterus ((Mapurito), Eremophilus mutisii (Capitán de la sabana) y Pimelodus pictus (Tigrito), han sido valoradas para su producción a nivel de laboratorio y se han evaluado algunos de sus aspectos reproductivos (Rodríguez-Pulido y Mojica-Benítez, 2005).

Ariopsis seemanni, es un bagre marino presente en la costa pacífica colombiana donde tradicionalmente los peces adultos de gran tamaño (mayor a $25 \mathrm{~cm}$ longitud total), han sido parte del consumo alimenticio de pobladores costeros. Sobre los peces de edad temprana de esta especie $(5-10 \mathrm{~cm}$ de longitud total), se ha presentado en las últimas dos décadas una alta presión pesquera sobre el recurso natural silvestre, debido a que son usados en acuarios, como peces ornamentales marinos, aclimatados a un ambiente dulceacuícola, y comercializados tanto a nivel nacional como internacional (Lasso et al., 2011). Los peces son capturados en las costas del Pacífico, comercializados nacionalmente en ciudades capitales (Cali, Medellín, entre otras) y exportados principalmente por vía aérea desde Bogotá a otros países. Esta especie en Colombia ha sido priorizada como una especie en la cual hay que desarrollar investigaciones debido a la potencialidad que tiene para actividades acuícolas (MADR y IICA, 2011).

Son pocas las investigaciones encontradas sobre Ariopsis seemanni. Estas se refieren principalmente a aspectos biológicos y ecológicos (Ortega, 1996), filogenéticos (Betancur et al., 2007), nutricionales (Dallos-Rodríguez, 2007; Lamouroux-López, 2014), morfológico relacionado con el encéfalo (Londoño y Hurtado-Giraldo, 2010), el tracto digestivo (GómezRamírez et al., 2010), el nervio óptico (Castañeda-Cortés et al., 2013), e histológico relacionado con el ojo (Tovar-Bohórquez et al., 2013).

Realizar experiencias encaminadas a la reproducción inducida de A. seemanni, llegan a ser herramientas hacia una alternativa para la producción de juveniles, dadas las condiciones reproductivas particulares relacionadas con esta especie. Así mismo se plantea la hipótesis, que peces tratados con HGC tendrán una mejor respuesta en el desarrollo gonadal en términos de cantidad de oocitos en maduración final y ovulación, con respecto a aquellos tratados con EPC. El presente ensayo evaluó el efecto de dos tratamientos hormonales con gonadotropina coriónica humana (HGC) y dos tratamientos hormonales con extracto pituitario de carpa (EPC), y sus efectos sobre la cantidad de oocitos en maduración final y el desove en Ariopsis seemanni.

\section{Materiales y métodos}

Área de estudio. La investigación se realizó en las instalaciones de la estación acuícola Bahía Málaga de la AUNAP ubicada en los $3^{\circ} 55^{\prime}$ Lat. $\mathrm{N}$ y los $77^{\circ} 21^{\prime}$ Long. O (Cantera et al., 2014), en el corregimiento de Juanchaco, jurisdicción del municipio de Buenaventura, caracterizada por una temperatura ambiental entre 
26 y $28^{\circ} \mathrm{C}$, humedad relativa del $90 \%$ y una pluviosidad de $9.000 \mathrm{~mm}$ (PNNC, 2014).

Área experimental. En $140 \mathrm{~m}^{2}$, se adecuó un sistema con abastecimiento de agua marina y aireación constantes, donde se ubicaron 24 tanques plásticos de 100 $\mathrm{L}$ con tapa, usados como contendores en cada una de las unidades experimentales para cada una de las parejas de peces (Figura 1).

Material biológico. Para el experimento se utilizaron 24 parejas (hembra y macho), que fueron obtenidos en finales del mes de enero y capturados del medio natural en la Quebrada Valencia, al interior del caserío de La Plata (4 $02 ` 16$ Lat. N y $77^{\circ} 13 ` 47$ Long. O) (Figura 2), colectados por pescadores artesanales de la región, utilizando anzuelos, chinchorro y atarraya. Estos peces fueron transportados en lancha una hora hasta la estación acuícola de la Aunap en Bahía Málaga. Al momento de la recepción de los peces, se verificaron si había presencia/ausencia de heridas en la piel,

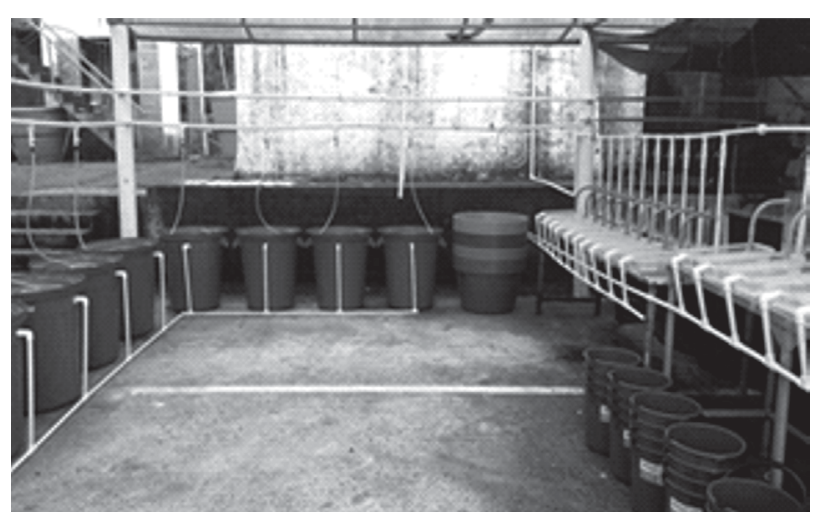

Figura 1. Área experimental para ensayo de reproducción inducida en Ariopsis seemanni. daños corporales, mutilaciones y movilidad natatoria en los peces.

Los procedimientos metodológicos a nivel de laboratorio que involucraron organismos vivos como peces, los cuales están relacionados con la manipulación, mantenimiento, observación, tratamiento, sacrificio y otros, tomaron en consideración el cumplimiento bioético de cuidado y bienestar animal, para el uso de organismos animales con propósitos de investigación, docencia y evaluación (CCAC, 2005; NRC. 2011).

Los peces obtenidos e identificados sexualmente, se ubicaron por sexo, en dos tanques de $2500 \mathrm{~L}$ cada uno (Figura 3) para facilitar su manipulación, durante 24 horas se mantuvo un flujo continuo $(6,6 \mathrm{~L} / \mathrm{min})$ de agua marina y aireación moderada, con el propósito de reducir el estrés por captura y transporte. Luego de este periodo, se procedió al registro de medidas biométricas en longitud y peso de cada reproductor. Para la manipulación de los peces, se utilizó como anestésico aceite de clavo de olor (LA-TOUR®) a una concentración de $10 \mathrm{ppm}$. La longitud total de cada reproductor se registró mediante una regla $(0-100 \mathrm{~cm})$ y el peso con una balanza digital (Kern modelo PCB, $1.0 \mathrm{~g}$ sensibilidad $0.1 \mathrm{~g}$ ). Se colocaron en proporción de 1:1 un macho y una hembra en cada unidad experimental.

Los peces utilizados tuvieron un peso promedio de $263.6 \pm 42.2$ g y $174.4 \pm 30.4$ g y una longitud total promedio de $25.31 \pm 1,28$ y $22.21 \pm 0.97 \mathrm{~cm}$, para hembras y machos, respectivamente.

Inducción hormonal. Tomando en consideración que para esta especie, no es viable realizar biopsias ováricas para extraer oocitos y determinar el estado exacto de desarrollo en ellos; así como también que no exis-
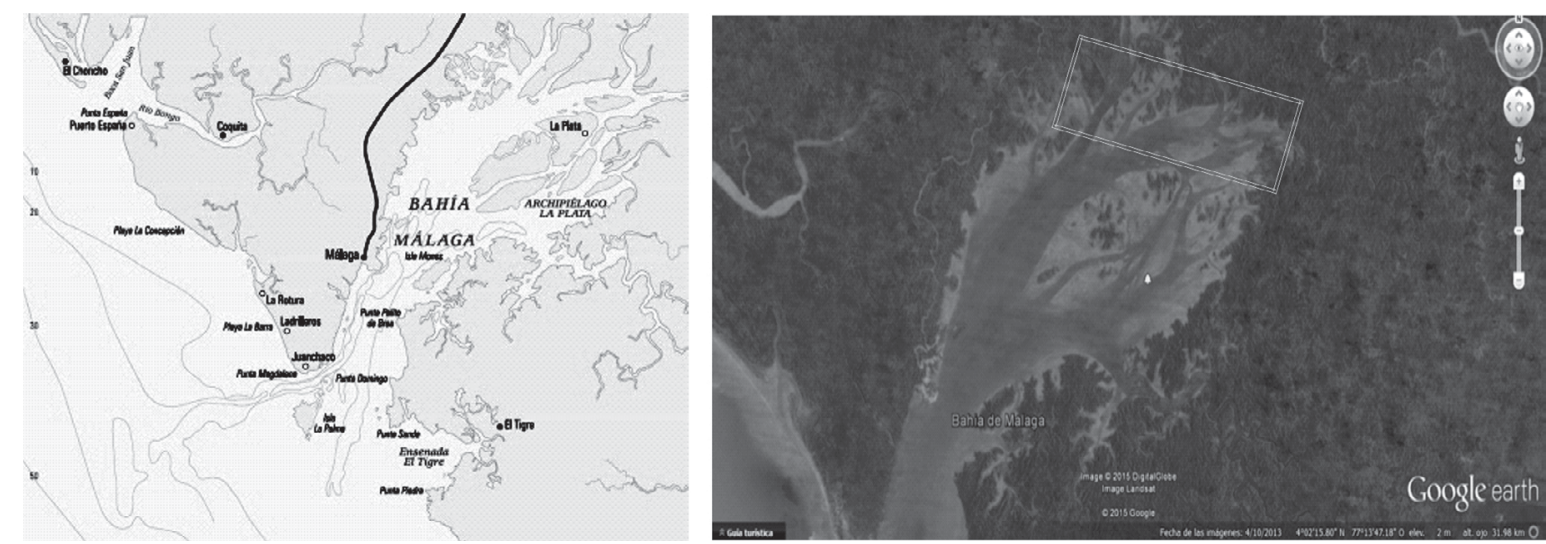

Figura 2. Zona (recuadro amarillo) de captura de peces de Ariopsis seemanni. Fuente: Google Maps, Google earth. 

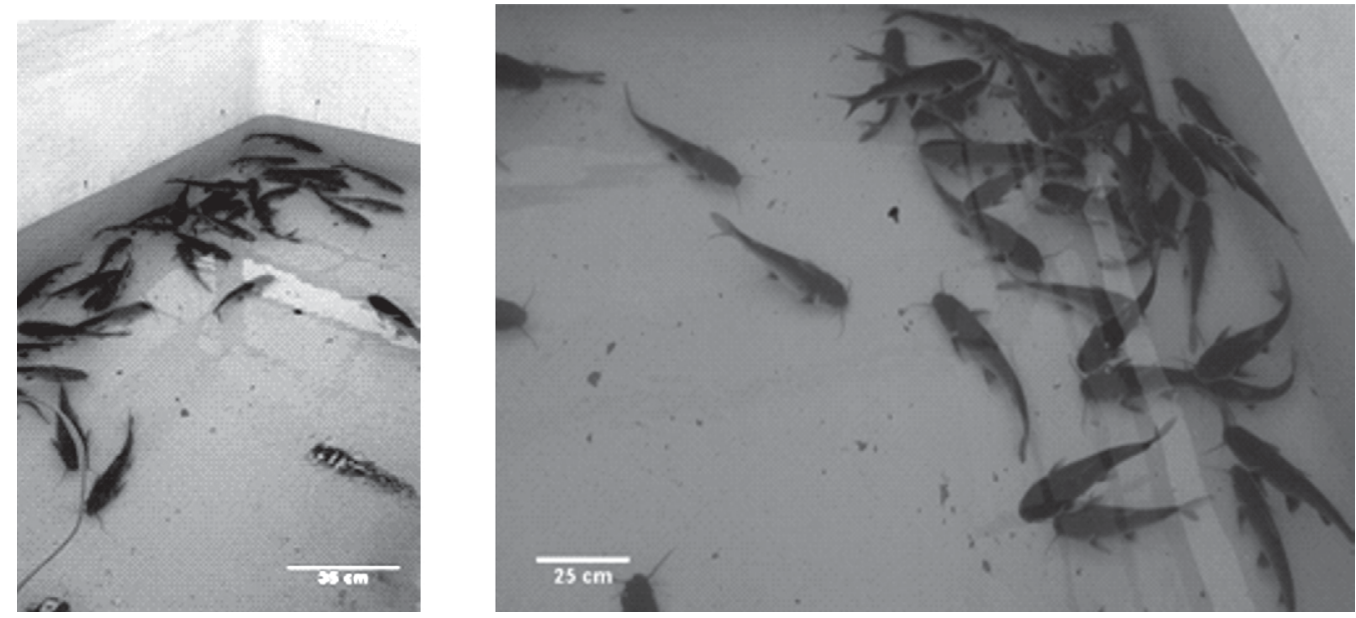

Figura 3. Peces adultos de Ariopsis seemanni separados por sexos: machos (Izquierda), hembras (Derecha)

te el referente científico indexado, acerca del estado del desarrollo de los oocitos, asociado directamente al grado de prominencia o volumen de la carnosidad presente en la base de las aletas pélvicas en las hembras de Ariopsis seemanni; (aspecto morfológico particular reconocido por algunos investigadores de la presencia de esta carnosidad en las épocas de reproducción y estados de madurez sexual de las hembras), al inicio del experimento, el estado de madurez en las hembras, se estimó de manera indirecta considerando el tamaño de la carnosidad en la base de las aletas pélvicas (figura 4).

Se plantearon cuatro tratamientos hormonales: $\mathrm{T}_{1}: 5$ mg EPC/kg; T2: 7 mg EPC/kg; T3: 2000 UI HGC/kg y T4: 1000 UI HGC $/ \mathrm{kg}$. Con base en el peso de cada reproductor, se determinó la cantidad de cada hormona a aplicar. En las hembras se aplicó una dosis preparatoria y doce horas después, una dosis final; en los machos se administró la mitad de la dosis total en cada trata- miento, suministrada cuando se aplicó la dosis final en hembras. Se utilizó un método de tratamiento hormonal invasivo agudo por inyección (Alvarez-Lajonchére y Hernández Molejón, 2001). Las dosis hormonales fueron inyectadas en la base de la aleta pélvica de los peces (figura 4), utilizando jeringas plásticas para insulina $(1 \mathrm{ml} \times 31 \mathrm{G} \times 8 \mathrm{~mm})$. Para corroborar que se presentó ovulación y/o desove en las hembras, se tomó en consideración el concepto propuesto por Woynarovich y Horváth, (Woynarovich y Horváth, 1980), en donde manifiestan que la ovulación comienza con la desaparición de la capa folicular del oocito celular, la concentración de los cromosomas y termina con la primera división meiótica. Al mismo tiempo, el folículo, que mantiene los huevos sujetos a la pared del ovario se rompe, se disuelve parcialmente y los oocitos caen a la cavidad ovárica. Y el desove, se comprende como la expulsión de los oocitos, en donde la masa de óvulos puede ya fluir libremente por el poro genital. Cada una de las hembras inducidas hormonalmente, fue sa-
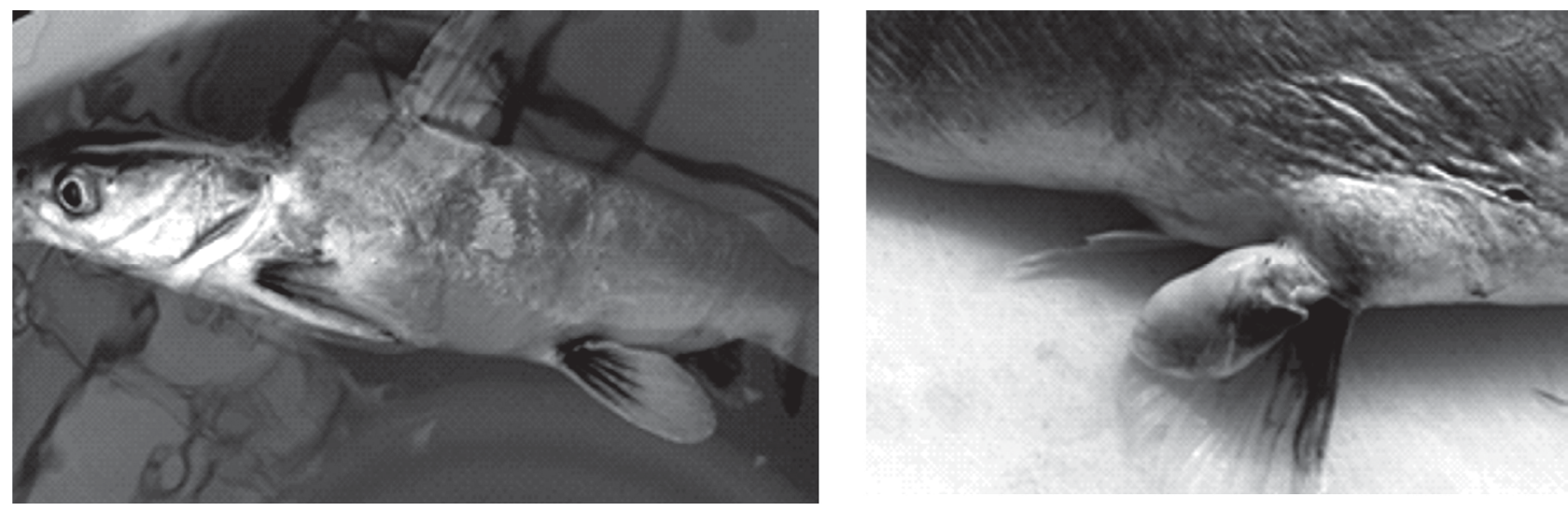

Figura 4. Aleta pélvica en Ariopsis seemanni (Izquierda). Carnosidad en aleta pélvica (Derecha) 
crificada por enfriamiento (usando hielo en el agua) y les fue practicada una disección post mortem, al final del experimento. La ausencia de desove espontáneo de las hembras se verificó con base en la no presencia de huevos en cada uno de los tanques de las unidades experimentales y por la ausencia de huevos en la cavidad bucal de cada macho potencialmente incubante revisado (Segura-Berttolini y Mendoza-Carranza, 2013). La disección post mortem, permitió posteriormente identificar el estado de madurez sexual en las hembras, utilizando los criterios planteados en la escala de madurez gonadal propuesta por Nikolsky (1963).

Para estimar el grado de desarrollo de los oocitos se consideró el tamaño y coloración; determinando: oocitos pequeños (3-5 mm) de color blanco, como oocitos inmaduros y oocitos grandes (> $12 \mathrm{~mm}$ ) color diferente a blanco, como oocitos en maduración final. Debido a que no hay referente indexado para la clasificación del tipo de oocitos en maduración final en esta especie, en este trabajo se planteó la siguiente clasificación: Oocitos tipo I. Oocitos en estado de maduración, de coloración amarillo - anaranjado y alta vascularización periférica; Oocitos tipo II. Oocitos en maduración, de coloración amarillo - verde, baja vascularización periférica; Oocitos tipo III. Oocitos, de coloración verde claro traslucido, escasa vascularización periférica. Tomando en consideración la cantidad de estos oocitos en maduración, se estimó la fecundidad promedio; se estimó el índice de ovulación, mediante el porcentaje de la relación entre la cantidad de hembras que presentaron ovulación respecto de la cantidad de hembras inducidas hormonalmente.

Monitoreo calidad del agua. En la fase de inducción hormonal fueron monitoreados parámetros fisicoquímicos en el agua: temperatura promedio, 28.43 \pm 0.36 ${ }^{\circ} \mathrm{C}$, medida un termómetro Brixco $\left(0-50{ }^{\circ} \mathrm{C}\right.$, sensibilidad $\left.1^{\circ} \mathrm{C}\right)$; concentración promedio de oxígeno disuelto (OD), $7.16 \pm 0.06 \mathrm{mg} / \mathrm{L}$, y porcentaje (\%) promedio de saturación, $80.2 \pm 0.006 \%$, medidos con una sonda paramétrica $\mathrm{YSI}$, modelo 550 ${ }^{\mathrm{a}}$; salinidad promedio 28.8 \pm 0.02 UPS, medida con un salinómetro refractómetro Atago ${ }^{\circledR}$ modelo $\mathrm{S} 10 \alpha$, y el flujo promedio de agua, $0.97 \pm 0.046 \mathrm{~L} / \mathrm{min}$, el cual se determinó con mediciones de aforo de caudal ( $\mathrm{L} / \mathrm{min})$.

Diseño experimental. Se utilizó un diseño de clasificación simple, con una distribución completamente al azar (DCA), con cuatro tratamientos y seis réplicas por tratamiento, para u $\mathrm{n}$ total de 24 unidades experimentales asociadas al modelo contrastadas al efecto de los tratamientos. Se utilizó el programa Statgraphics ${ }^{\circledR}$ Centurión XV versión 15.02.5, que permitió realizar análisis de varianza paramétrica de efectos fijos (ANO-
VA) simple, los análisis efectuados se realizaron a un nivel de confiabilidad al 95\%. La aleatoriedad de los datos se verificó, mediante la comprobación de los supuestos de homocedasticidad (Levene's) y prueba de normalidad (Kolmogorov-Smirnov).

\section{Resultados}

La Figura 5 presenta algunos de los rasgos externos de madurez sexual que se reconocieron en hembras de Ariopsis seemanni. La tabla 1 presenta, respuesta a ovulación y al desove en cada hembras de cada tratamiento, signo (-) denota evento no ocurrido, signo (+) evento ocurrido, cantidad y tipo de oocitos en maduración final. La Figura 6 presenta la cantidad de oocitos en maduración en cada uno de las hembras en cada tratamiento. Al final del experimento, en la Figura 7 se pueden observar los diferentes tipos de oocitos encontrados en estado de maduración.

\section{Discusión}

Se reconocieron rasgos externos de madurez sexual en las hembras considerando proyección y enrojecimiento de la papila urogenital y presencia de carnosidad en la aleta pélvica (Woynarovich y Horváth, 1980; Acevedo y Ángel, 1999; Chaparro, 1994). La inflamación de la papila urogenital observada en las hembras de $A$. seemanni, es un rasgo que también ha sido reportado en hembras maduras de Bagre marinus (Caballero-Chávez, 2013); en hembras de 19 cm LT de A. bonillai, donde se reconoce este rasgo y donde se presenta una carnosidad triangular en la aleta pélvica, que se torna muy agrandada y enrojecida durante el estado de madurez sexual (Galvis-Cortes, 1983; OlayaNieto et al., 2007).

Aunque la condición inicial de madurez en las hembras de A. seemanni, fue asumida de manera indirecta, comprendiendo que los rasgos externos no son criterios de garantía absoluta de la determinación de un desarrollo oocitario exacto (Zanuy y Carrillo, 1987), se reconoce, la necesidad de haber determinado la posición del núcleo en los oocitos que hubiesen podido haber sido extraídos de la gónada (Acevedo y Ángel, 1999), mediante biopsia ovárica (Zanuy y Carrillo, 1987); que para el caso de A. seemanni, no es viable, o recomendable debido al gran tamaño (> $12 \mathrm{~mm}$ ) en los oocitos, los que al tratar de ser extraídos mediante una cánula de un diámetro de 15-20 mm, causarían daño y lesiones en el oviducto en las hembras, posiblemente conllevando traumas que pueden bloquear los procesos endocrinos necesarios para maduración y el desove y en caso extremo causar la muerte en las 


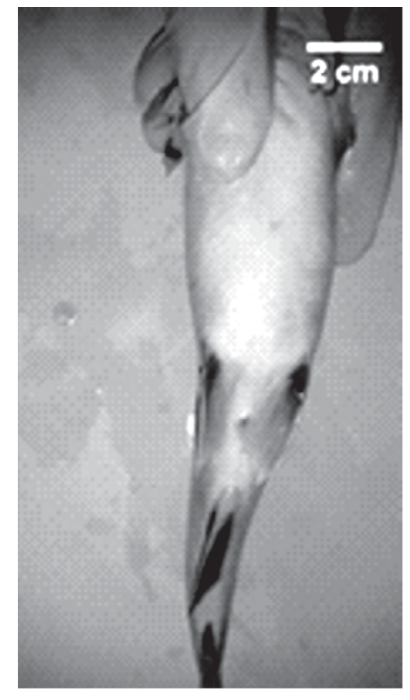

A

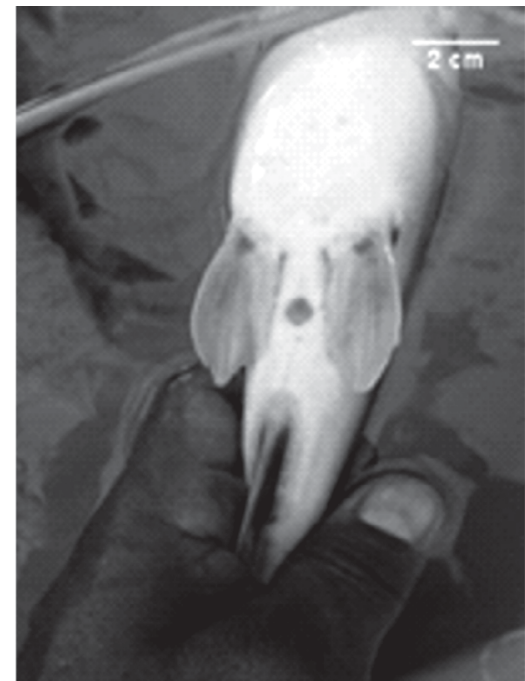

B

Papila urogenital: A. no proyectada. B. proyectada y enrojecida

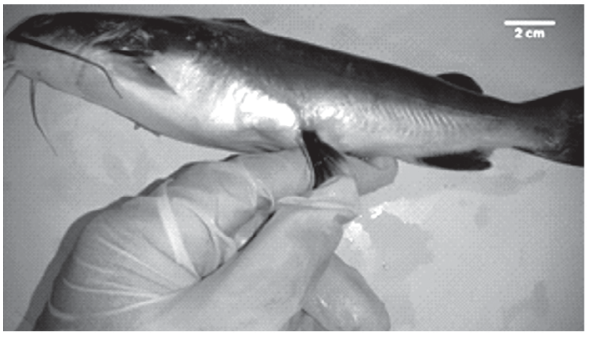

C

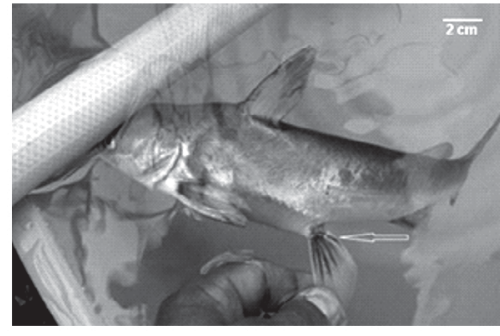

D

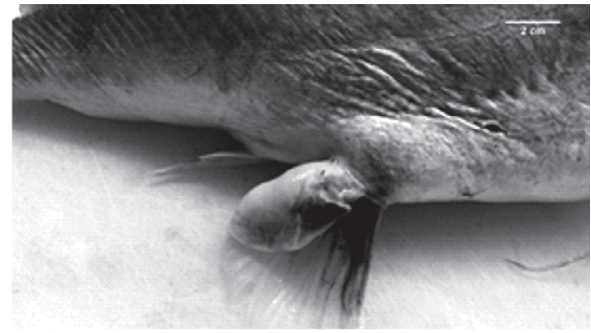

E

Volumen de carnosidad en aleta pélvica: C. ausente. D. iniciando. E. desarrollada

Figura 5. Signos externos de madurez sexual en hembras de Ariopsis seemanni.

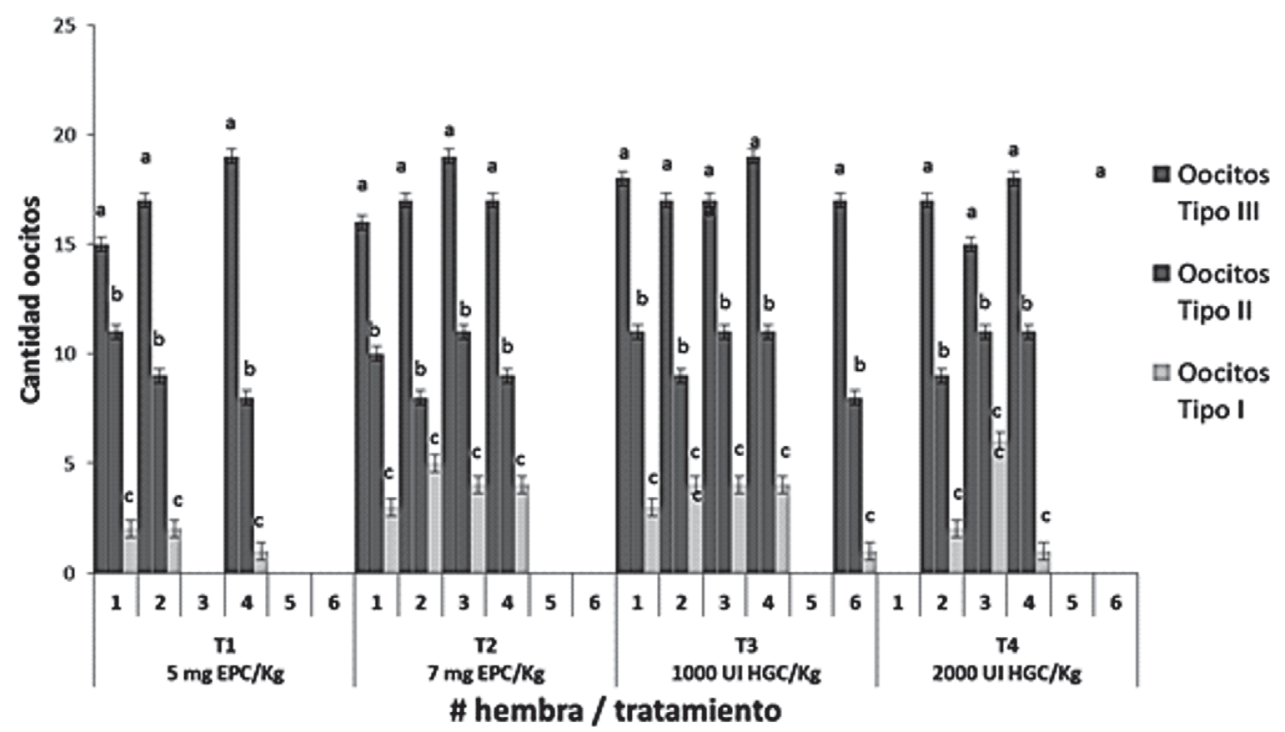

Barras de igual color con igual letra, denotan la no existencia de diferencias estadísticas significativas

Figura 6. Cantidad de oocitos en maduración en cada hembra de Ariopsis seemanni en cada tratamiento. 
Tabla 1. Respuesta reproductiva en hembras de Ariopsis seemanni, cantidad de oocitos en maduración en un ensayo de reproducción inducida $(n=24)$.

\begin{tabular}{|c|c|c|c|c|c|c|c|}
\hline Tratamiento & Répl. & \multicolumn{2}{|c|}{ Ovul. Desov. } & $\begin{array}{c}\text { Oocitos } \\
\text { Tipo I }\end{array}$ & $\begin{array}{c}\text { Oocitos } \\
\text { Tipo II }\end{array}$ & $\begin{array}{l}\text { Oocitos } \\
\text { Tipo III }\end{array}$ & $\begin{array}{c}\text { Total Oocitos } \\
\text { Mad. }\end{array}$ \\
\hline \multirow{6}{*}{$\begin{array}{c}\mathrm{T}_{1} \\
5 \mathrm{mg} \mathrm{EPC} / \mathrm{kg}\end{array}$} & 1 & + & - & 2 & 11 & 15 & 28 \\
\hline & 2 & + & - & 2 & 9 & 17 & 28 \\
\hline & 3 & - & - & 0 & 0 & 0 & 0 \\
\hline & 4 & + & - & 1 & 8 & 19 & 28 \\
\hline & 5 & - & - & 0 & 0 & 0 & 0 \\
\hline & 6 & - & - & 0 & 0 & 0 & 0 \\
\hline \multirow{6}{*}{$\begin{array}{c}\mathrm{T}_{2} \\
7 \mathrm{mg} \mathrm{EPC} / \mathrm{kg}\end{array}$} & 1 & + & - & 3 & 10 & 16 & 29 \\
\hline & 2 & + & - & 5 & 8 & 17 & 30 \\
\hline & 3 & + & - & 4 & 11 & 19 & 34 \\
\hline & 4 & + & - & 4 & 9 & 17 & 30 \\
\hline & 5 & - & - & 0 & 0 & 0 & 0 \\
\hline & 6 & - & - & 0 & 0 & 0 & 0 \\
\hline \multirow{6}{*}{$\begin{array}{c}\mathrm{T}_{3} \\
1000 \mathrm{UI} \mathrm{HGC} / \mathrm{kg}\end{array}$} & 1 & + & - & 3 & 11 & 18 & 32 \\
\hline & 2 & + & - & 4 & 9 & 17 & 30 \\
\hline & 3 & + & - & 4 & 11 & 17 & 32 \\
\hline & 4 & + & - & 4 & 11 & 19 & 34 \\
\hline & 5 & - & - & 0 & 0 & 0 & 0 \\
\hline & 6 & + & - & 1 & 8 & 17 & 26 \\
\hline \multirow{6}{*}{$\begin{array}{c}\mathrm{T}_{4} \\
2000 \mathrm{UI} \mathrm{HGC} / \mathrm{kg}\end{array}$} & 1 & - & - & 0 & 0 & 0 & 0 \\
\hline & 2 & + & - & 2 & 9 & 17 & 28 \\
\hline & 3 & + & - & 6 & 11 & 15 & 32 \\
\hline & 4 & + & - & 1 & 11 & 18 & 30 \\
\hline & 5 & - & - & 0 & 0 & 0 & 0 \\
\hline & 6 & - & - & 0 & 0 & 0 & 0 \\
\hline
\end{tabular}

Répl.= Réplica; Ovul.= Ovulación; Desov. = Desove; Mad.= Maduros.

hembras. Se logró identificar estado IV de madurez sexual en las hembras al final del experimento (Nikolsky, 1963).

En los sacos ováricos de $A$. seemanni, se pudieron observar oocitos en diferentes estados de desarrollo (tamaño, coloración y posición en el saco ovárico). Oocitos en maduración, hacia el extremo posterior del saco ovárico y oocitos inmaduros en la región anterior. La presencia de oocitos en diferente estado de desarrollo podría hacer considerar: 1) Un desarrollo diferencial o asincrónico de los folículos reproductivos. Esta condición asincrónica, estaría acorde al concepto planteado años atrás para la especie (Ortega, 1996). 2) Un desarrollo sincrónico por grupos de oocitos, que conllevaría a un proceso de reproducción durante varios días, teniendo desoves parciales. Este concepto podría considerarse, como estrategia reproductiva que favorece la supervivencia de los alevinos; tomando en consideración el planteamiento que refiere la capacidad bucal que tienen los machos de algunos Áridos en los procesos de incubación de huevos fecundados. Un caso contrario es lo reportado por Amezcua y Muro-Torres (2012), para Occidentarius platypogon, otra especie de bagre, donde encontraron una condición de madurez gonadal, que les permitió determinar un 

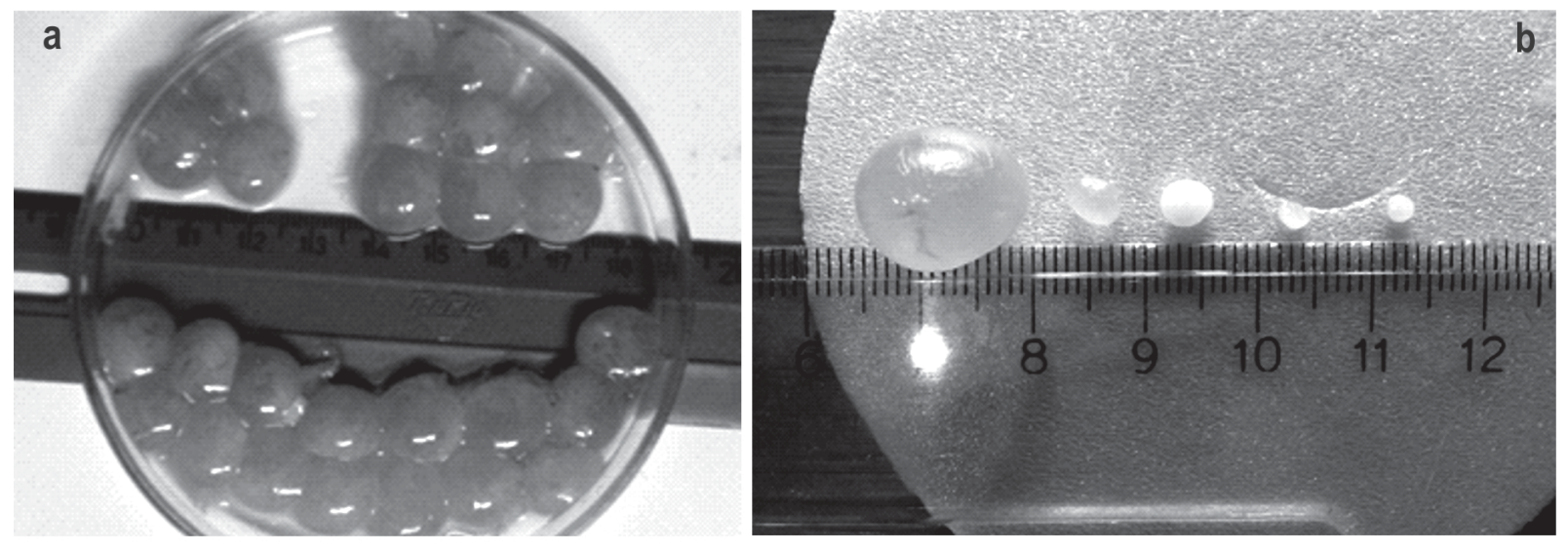

Figura 7. a) Oocitos en estado de maduración; b) oocitos inmaduros color blanco.

desarrollo sincrónico de oocitos y un proceso de desove anual.

La fecundidad media encontrada en las hembras de A. seemanni fue de $3.07 \pm 1.02$ para oocitos en maduración: tipo I, de $9.80 \pm 1.86$ para oocitos tipo II y de $17.20 \pm 1.26$ para oocitos tipo III; la fecundidad promedio total fue de $30.07 \pm 2.34$ oocitos en maduración final (considerando las 15 hembras en las que se encontraron estos tipos de oocitos). Valores cercanos son reportados por Acero, 2000 (en OlayaNieto et al., 2007) en A. bonillai con una fecundidad media de 36 oocitos. Para el Bagre marinus, se reportaron cantidades de 21 a 33 ovocitos hidratados y un máximo de 62; este último valor en hembras maduras de 350 a $555 \mathrm{~mm}$ de longitud y 351 a 1980 g de peso (Mendoza-Carranza y Hernández-Franyutt, 2005). Podría considerarse, de manera preliminar, que $A$. seemanni es una especie de fecundidad promedio entre las especies de áridos de este género.

Por otra parte, el diámetro promedio global de los oocitos de A. seemanni, fue de 13.2 $\pm 3.6 \mathrm{~mm}$. Estos tamaños son similares a los reportados para A. bonillai, por Toro y Villa (1983) entre 1-2 cm; por Chaparro et al., (2002) entre 0.9-1.6 cm; por Olaya-Nieto, et al., (2007) entre 0.8-1.6 cm y también por Olaya-Nieto et al., (2007) con un promedio de $1.2 \pm 0.13 \mathrm{~cm}$

Según los resultados relacionados con fecundidad y tamaño de los oocitos, en A. seemanni, se conservan los planteamientos de Yáñez-Arancibia (Olaya-Nieto et al., 2007), acerca de los bagres marinos donde se manifiesta una aparente baja fecundidad, en un sentido estricto, con base al tamaño de sus oocitos, pero donde biológicamente existen mecanismos que aseguran una alta supervivencia de huevos y larvas, huevos grandes en tamaño y reservas de vitelo y una protección de huevos y larvas, gracias a la incubación bucal de los machos.

Las medidas en longitud y peso en las hembras de $A$. seemanni, muestran ejemplares entre 28 a $33 \mathrm{~cm}$ y entre 189 a 357 g, en estado de madurez sexual. Longitudes similares se reportan en hembras de otros bagres marinos: $25.2 \mathrm{~cm}$ de longitud furcal en hembras maduras de Bagre marinus (Caballero-Chávez, 2013); para Occidentarius platypogon, una longitud de madurez sexual de $35 \mathrm{~cm}$ (Amezcua y Muro-Torres, 2012); en A. bonillai, la talla de primera madurez sexual reportada entre 22.0 y 24.0 cm LT (Olaya-Nieto et al., 2007).

La maduración en oocitos se pudo verificar al reconocer y diferenciar oocitos en diferente color y tamaño respecto a oocitos pequeños inmaduros de color blanco (Figura 7). La ovulación se observó encontrando oocitos en maduración en el saco ovárico, que no tenían conexión con la pared del ovario. En todos los tratamientos hormonales, se tuvieron hembras que presentaron oocitos en estado de maduración final, solamente nueve hembras inducidas hormonalmente, no presentaron este tipo de oocitos en maduración final. Los resultados de los análisis estadísticos efectuados permiten inferir que no existieron diferencias estadísticamente significativas (figura 6), entre los tratamientos utilizados frente a la cantidad de cada uno de los tipos de oocitos en estado de maduración final ovulados $(P$ Valor: $p=0,60 ; p=0,59 ; p=0,34$; para oocitos Tipo III, II y I respectivamente). Se realizaron análisis de varianza para analizar si pudiese existir efecto del peso o la longitud de las hembras en cada uno de los tratamientos, llegando a determinar que tampoco existieron diferencias estadísticamente significativas al respecto (Peso: $P$ valor $=0,16$; Longitud: $P$ valor $=0,49$ ). 
No se obtuvieron desoves espontáneos en las hembras inducidas; probablemente este evento se puede asociar a varios sucesos: haber utilizado peces no adaptados durante varios meses a condiciones de laboratorio, que las dosis de hormona utilizada en los tratamientos, aunque produjeron ovulación (índice de ovulación del 50\% al $66,67 \%$ con EPC y del $50 \%$ al $83,33 \%$ con HGC), posiblemente pudiesen haber tenido un efecto insuficiente sobre el desove, y que el mismo proceso de desove estuviese influenciado adicionalmente por estímulos exógenos, a los que se tuvieron durante el experimento; por ejemplo haber colocado refugios artificiales en los tanques, disponer de un sustrato adicional diferente (Tucker, 1966), haber utilizado una proporción macho a hembra mayor, que produjera mayor disponibilidad de feromonas en el medio, posibilitando un desove grupal más eficiente, que en parejas de peces aisladas (Mylonas et al., 2010), una condición de baja salinidad que pudiese haber mejorado procesos fisiológicos similares a ambientes naturales. Los planteamiento expuestos, hacen necesario considerar en la reproducción inducida en A. seemanni, la inclusión de condiciones ambientales abióticas y factores externos de manejo como factores importantes, adicionales al agente hormonal (Carrillo et al., 2009). En este sentido, algunos de los valores de calidad de agua registrados durante el experimento, estuvieron dentro del rango de confort reportados para reproducción de especies tropicales (Yu et al., 2008).

Aunque existen concentraciones de la HGC con la cuales se han logrado desoves en especies como Lutjanus guttatus, Lutjanus campechanus, Morone saxitilis, Dicentrarchus labrax entre algunas, el uso de HGC se ha generalizado; sin embargo, su molécula es muy diferente a la de los peces, por lo que no ha sido efectiva o eficiente en algunas especies con las dosis sugeridas y requeridas lo que puede ocasionar utilizar altas cantidades, con márgenes de seguridad muy estrechos, por lo que se pueden provocar sobredosis que a menudo producen desoves parciales, obstrucción del poro genital, entre otros, con resultados negativos, tanto por la mala calidad de los huevos, como causar daños e incluso mortalidad en los reproductores (Alvarez-Lajonchére y Hernández, 2001). La cantidad de hormona utilizada en cada uno de los tratamientos de esta investigación, se planteó como dosis punto de partida, considerando los posibles efectos, mencionados anteriormente. Se deben implementar prácticas con nuevas dosis y otras presentaciones hormonales que puedan inducir la ovulación.

\section{Conclusión}

Las concentraciones de 5 y $7 \mathrm{mg}$ de EPC/kg y de 1000 y de 2000 UI de HGC/kg, empleadas durante el ex- perimento lograron inducir ovulación de oocitos en hembras de $A$. seemanni en estado de madurez sexual capturadas del medio natural, No se lograron desoves espontáneos en las hembras inducidas, evento que pudo estar influenciado por la ausencia de otros factores exógenos determinantes a los manejados durante la investigación. La presente investigación se reporta como el primer trabajo de reproducción inducida para la especie.

\section{Agradecimientos}

A la Autoridad Nacional de Acuicultura y pesca AUNAP, por facilitar las instalaciones para la fase experimental de la presente investigación

\section{Referencias}

Acevedo C, Angel F. 1999. Reproducción inducida e incubación del bagre pintado (Pseudoplatystoma fasciatum) (Linnaeus, 1766) y barbudo (Pimelodus) Palmira, Valle del Cauca, Colombia.

Alvarez-Lajonchère L, Hernández M. 2001. Producción de juveniles de peces estuarinos para un centro en América Latina y el Caribe: diseño, operación y tecnologías. (D. C. Browdy, Ed.) Baton Rouge, Louisiana, Estados Unidos de América - USA: The World Aquaculture Society.

Amezcua F, Muro-Torres V. Biología reproductiva del bagre cominate Occidentarius platypogon (Pisces: Ariidae) en el sureste del golfo de California. Latin American. J Aquac Res. 2012;2(40):428-434.

Betancur R, Acero, A, Bermin E. Systematics and biogeography of New World sea catfishes (Siluriformes: Ariidae) as inferred from mitochondrial, nuclear, and morphological evidence. Mol Phylogenet Evol. 2007;45(1):339-357.

Caballero-Chávez V. Madurez y reproducción de bagre bandera Bagre marinus en el sudeste de Campeche. Ciencia Pesquera. 2013;21(2):13-19.

Cantera JR, Escarria E, Mejia L, Medina D. Distribución y densidad de ictioplancton en el Estuario de Bahía Málaga, pacífico colombiano (septiembre de 2009-febrero de 2010). Bol Investig Mar Costeras. 2014;43(1):107-119.

Carrillo M, Zanuy S, Bayarri M. 2009. El control ambiental de la reproducción de los peces con especial referencia al control del ciclo sexual, de la pubertad y de la precosidad. En C. M. Adrián, La Reproducción de los Peces - aspectos básicos y sus aplicaciones en acuicultura (Serie: Publicaciones Científicas y Tecnológicas de la Fundación Observatorio Español de Acuicultura ed., págs. 173-233). Madrid, España : Fundación Observatorio Español de Acuicultura.

Castañeda-Cortés D, Tovar-Bohorquez O, Hurtado-Giraldo H. Estudio Morfométrico del Nervio Óptico de Tiburoncito (Ariopsis seemanni). Int J Morphol. 2013;31(1):184-188.

CCAC, 2005. Guidelines on: the care and use of fish in research, teaching and testing, Canadian Council on Animal Care. ISBN: 0-919087-43-4. 94 Pag. 
Chaparro, N. 1994. Reproducción artificial y manipulación genética en peces. Barranquilla, Colombia: Editorial Mejoras...84 Pag.

Chaparro N, Gaitán S, A. Hernández. 2002. Incubación, larvicultura y alevinaje del bagre estuarino Ariopsis bonillai (Miles, 1945) en cautiverio, Santa Marta, Caribe colombiano. Informe técnico. Santa Marta: Universidad del Magdalena-Colciencias

Cruz-Casallas P, Velasco-Santamaría YM, Medina-Robles M. Manejo hormonal de la función reproductiva de peces tropicales bajo condiciones de cautiverio. Revista electrónica en producción acuícola Universidad de Nariño. 2006;2(2).

Dallos-Rodríguez J. 2007. Efecto de la suplementación con ácido ascórbico sobre el crecimiento y la supervivencia en juveniles de Hexanematichthys seemanni (Günther, 1864). VI Seminario Internacional de Acuicultura, Bogotá, 2007. Bogotá, Colombia.

Galvis-Cortes, O. 1983. Los áridos de la Ciénaga Grande de Santa Marta.. (Universidad-Nacional-de-Colombia,, Ed.) Bogotá., Colombia, 186 pp .

Gómez-Ramírez E, Tovar-Bohórquez MO, Obando-Bulla MJ, Hurtado-Giraldo $\mathrm{H}$. Estudio histológico del tracto digestivo del pez Ariopsis seemanni (Ariidae). Rev Fac Cienc B UMNG. 2010;6(2):216-225.

Lamouroux-Lopez SL. Efecto de nivel de proteína sobre el desempeño productivo del tiburoncito Ariopsis seemanni. Orinoquía. 2014;18(2):212-217.

Lasso C, Agudelo-Córdoba E, Jiménez-Segura L, Ramírez-Gil H, Morales-Betancourt M, Ajiaco-Martínez R, et al. 2011. I. Catálogo de los recursos pesqueros continentales de Colombia (Instituto de Investigación de Recursos Biológicos Alexander von Humboldt (IAvH) ed., Vol. Serie Editorial Recursos Hidrobiológicos y Pesqueros Continentales de Colombia). Bogotá, Colombia.

Londoño C, Hurtado-Giraldo H. Estudio preliminar morfológico y morfométrico de encéfalo del pez tiburoncito, Ariopsis seemanni, (Pisces: Ariidae). Univ Sci. 2010;15(2):101-109.

MADR, \& IICA. 2011. Agenda nacional de investigación en pesca y acuicultura 2011-2012. Ministerio de Agricultura y Desarrollo Rural.Bogotá, Colombia:

Mendoza-Carranza M, Hernández-Franyutt A. Annual reproductive cycle of gafftopsail catfish, Bagre marinus (Ariidae) in a tropical coastal environment in the Gulf of Mexico. Hidrobiológica. 2005; 15(3):275-282.

Mylonas C, Fostier A, Zanuy S. Broodstock management and hormonal manipulations of fish reproduction. Gen Comp Endocrinol. 2010;165:516-534.
National Research Council of the National Academies. 2011. Guide for the care and use of laboratory animals. Eighth Edition. The national academies press. Washington. 246 Pag

Nikolsky GV. 1963. The Ecology of Fishes. Academic Press, NewYork, $352 \mathrm{pp}$

Olaya-Nieto CW, Segura-Guevara FF, Tordecilla-Petro G, Appeldoorn RS. 2007. Estimación de los parámetros biológicos básicos de peces comerciales del rio Sinú - II fase. Informe Final, Universidad de Córdoba. Lorica, Córdoba.

Ortega A. 1996. Aspectos de la biología y la abundancia de Arius seemanni (Pisces: Siluriformes: Ariidae) en un manglar de la bahía de Buenaventura (pacifico colombiano). Ed. Universidad del Valle, Cali, Valle del cauca, Colombia.

PNNC, 2014. Parques Nacionales de Colombia. Recuperado el 23 de 09 de 2015, de www.parquesnacionales.gov.co

Rodríguez-Pulido J, Mojica-Benítez H. 2005. Reproducción y manejo de silúridos en cautiverio. En INCODER, \& Universidad Nacional de Colombia, Reproducción de peces en el trópico. Bogotá, Colombia: Imprenta Nacional de Colombia, p. 105-122.

Segura-Berttolini E, Mendoza-Carranza M. La importancia de los machos del bagre bandera, Bagre marinus (Pisces: Ariidae), en el proceso reproductivo. Cienc Mar. 2013;39(1):29-39.

Toro M, Villa F.1983. Algunos aspectos de la historia de vida de Ariopsis bonillai en la Ciénaga de Tesca. Tesis de Biología Marina. Univ. Jorge Tadeo Lozano. Bogotá, Colombia

Tovar-Bohórquez M, Corchuelo-Chavarro S, Gómez-Ramírez E, Obando-Bulla M, Hurtado-Giraldo H. Histología y morfometría del ojo del pez de Ariopsis seemanni: Implicaciones en ecología visual. Hidrobiológica. 2013;23(3):420-430.

Tucker C. The ecology of channel catfish culture ponds in Northwest Mississipp. Rev Fish Sci. 1966;4(1):1-55.

Woynarovich E, Horváth L. 1980. The artificial propagation of warmwater finfishes - a manual for extension. FAO. FAO Fisheries and Aquaculture Department.

Yu C, Xing B, Xu L, Li D. 2008. Water quality management in intensive aquaculture in china. En: IFIP International Federation for Information Processing, Volume 259; Computer and Computing Technologies in Agriculture, Vol 2; Daoliang Li, (Boston: Springer);143-1252.

Zanuy S, Carrillo M. 1987. Reproducción en acuicultura. J. Espinosa de los Monteros, \& U. Labarta (Edits.). España. 